\title{
A Network Pharmacology-based Study of the Potential Mechanism of Bushenhuoxue Formula in Treating Osteoarthritis
}

\section{Xiong Wen}

The First School of Clinical Medicine, Southern Medical University https://orcid.org/0000-0001-50445516

\section{Cai Xianhua ( $\square$ harrisqis@126.com )}

\section{Research}

Keywords: BuShenHuoXue, formula, network pharmacology, Osteoarthritis, enrichment analysis, quercetin

Posted Date: August 3rd, 2020

DOI: https://doi.org/10.21203/rs.3.rs-48816/v1

License: (1) This work is licensed under a Creative Commons Attribution 4.0 International License. Read Full License 


\section{A network pharmacology-based study of the potential mechanism of}

BuShenHuoXue formula in treating osteoarthritis

Full names of all authors: Xiong Wen ${ }^{1}$, Cai Xianhua ${ }^{2}$

Department and institutional affiliations of the authors:

${ }^{1}$ The First School of Clinical Medicine, Southern Medical University

2 The First School of Clinical Medicine, Southern Medical University

Department of Orthopaedic Surgery, General Hospital of Central Theater Command

Telephone number of the author responsible for manuscript preparation: $+86-13607132282$

E-mail address to speed up contacts with authors : harrisqis@126.com

\section{Abstract}

Background: To investigate the potential mechanism underlying the efficacy of BuShenHuoXue (BSHX) formula on Osteoarthritis (OA) and its molecular mechanism. Materials and Methods: Data as for bioactive chemicals of individual herb in BSHX formula and their targets were collected from Traditional Chinese Medicine Systems Pharmacology database and OA-associated targets from Gene Expression Omnibus database, compound-disease target network and protein-protein interactions network were built, picturized and analyzed by Cytoscape. Gene Ontology and Kyoto Encyclopedia of Genes and Genomes pathway enrichment of key targets were carried out and analyzed to probe into the core pathway and their main functions further. The chondrocytes of SD rats were cultured in vitro, and $50 \mu \mathrm{g} / \mathrm{ml} \mathrm{IL-1} \beta$ was added to the chondrocytes to induce apoptosis. Different concentrations of quercetin were added to the experimental group and the apoptosis rate of chondrocytes, the difference of the expression of SELE, MMP2, and COL1 genes and their protein expression level were further detected. Results: A total of 104 candidate chemicals and 42 crossing targets were screened out. 
Leading target genes are PTGS2, NCOA2 and HSP90AA1, whereas quercetin and luteolin are principal ingredients. Potential pathways against OA are AGE-RAGE signaling pathway in diabetic complications, Relaxin signaling pathway, IL-17 signaling pathway, Tyrosine metabolism and Endocrine resistance. Our study showed that quercetin could inhibit the apoptosis of chondrocytes induced by IL-1 $\beta$, decrease SELE, MMP2 and COL1 mRNA expression, likewise decrease the expression of SELE, MMP2 and COL1 protein. Conclusion: This study investigated the bioactive chemicals, crossing targets and possible mechanisms of BSHX formula against OA by network pharmacology strategy, results suggests that quercetin in BSHX formula may target on SELE, MMP2, and COL1 genes and then inhibit the progression of OA through the AGE-RAGE signaling pathway in diabetic complications. By the mechanism of reducing the apoptosis rate of SD rat chondrocytes and down-regulation the expression of genes involved in inflammation, we made sure that quercetin as principal ingredient can protect the cartilage. In addition, the conclusion of this study still need to be confirmed by in vivo and vitro experiments.

Key words: BuShenHuoXue, formula, network pharmacology, Osteoarthritis, enrichment analysis, quercetin.

\section{Background:}

Osteoarthritis is a common chronic bone and joint disease. It occurs in all the joints of the body, such as hip, knee, shoulder, spine etc. Research shows that osteoarthritis afflicts more than 360 million people worldwide [1], and its morbidity incidence is increasing year by year [2]. 80 percent of the patients present exercise limitations and 25 percent physical disability [3]. Although scholars have conducted a large number of studies on osteoarthritis, the pathogenesis of osteoarthritis has been unclear [4]. So far, the pharmacological management of OA has targeted the symptoms, rather than the underlying etiological factors. Given the high morbidity rate of OA and the shortage of long-term 
effective pharmacological therapies, it is imperative to discover potential chemicals with ameliorative effects.

Traditional Chinese Medicine (TCM) is a general term for traditional medicines mainly derived from different natural medicines and herb products. It dates back from ancient China and has made significant contributions to human health[5,6]. With minimal side effects, it shows better prospect as complementary or alternative medicine for osteoarthritis. Furthermore, it shows remarkable effects in protecting articular cartilage $[7,8]$. In recent years, the advancement of analytical technologies and methodologies has greatly accelerated the research of TCM. In TCM theory, sustainable homeostasis maintenance and regeneration of bone and cartilage depend on sufficient essence of kidney and liver [9]. Deficient essence of kidney and liver together with blood stasis can ultimately lead to cartilage damage. BSHX formula, which consist of 10 main herbs (Glycyrrhiza uralensis, Rehmannia glutinosa, Eucommia ulmoides, Aconitum carmichaeli, Lycium barbarum, Cinnamomum cassia, Cornus officinalis, Prunus persica, Carthamus tinctorius, Dioscoreae opposite), has been used in practice for many years in OA treatment and yielded positive outcome [10, 11]. Besides, Wang[12] testified BSHX formula and proved that it could prohibit cartilage degradation in mouse OA model. While the unclear mechanism of TCM has hampered its clinical practice in the world, the therapeutically effect of BSHX on osteoarthritis remains to be further elucidated.

Network pharmacology(NP) is an burgeoning drug research strategy that studies the effects of drugs on diseases in a complex biological network and uses related gene databases to explore the mechanism [13]. NP accelerate the development of pharmacology and elucidates mechanisms of drug action by establishing a multi-layer network of disease-phenotype-gene-drug, which is of the same to TCM research [14].

In this study, a compound-disease target network was constructed with the methods of NP prediction. We tried to investigate the bioactive chemicals, crossing targets and possible mechanisms of BSHX against osteoarthritis by NP strategy involving multiple drug target prediction, protein2 
protein interaction network and enrichment analysis. A following experiment was conducted which validated the effects of bioactive ingredients for BSHX in the treatment of OA.

\section{Materials and Methods}

\subsection{Data mining: Screening out bioactive chemicals and targets in BuShenHuoXue formula}

Chemicals and targets of individual herbs (Glycyrrhiza uralensis, Rehmannia glutinosa Eucommia ulmoides, Aconitum carmichaeli, Lycium barbarum, Cinnamomum cassia, Cornus officinalis, Prunus persica, Carthamus tinctorius, Dioscoreae opposite) in BSHX Formula (Table 1) were mined from Traditional Chinese Medicine Systems Pharmacology Database (TCMSP: http://tcmspw.com/tcmsp.php), which is a systematic pharmacology database containing bioactive ingredients of Chinese herbal medicine and their corresponding targets [15]. The screening criteria of herbal medicine in TCMSP is Absorption, Distribution, Metabolism and Excretion (ADME) including oral bioavailability (OB), drug-likeness(DL), P450, etc.[14]. OB and DL represents proportion of drug absorbed into human circulation, the similarity between herbal ingredients and specific medicines respectively, suggesting that herbs are potentially therapeutic agents. $\mathrm{OB} \geq 30 \%$ and $\mathrm{DL} \geq 0.18$ are chosen as screening conditions for potential bioactive chemicals and related-targets by Strawberryperl software (https://www.perl.org, ver.5.30.1.1).

\subsection{Searching and screening for potential Osteoarthritis targets}

OA-associated targets were acquired from Gene Expression Omnibus(GEO: https://www.ncbi.nlm.nih.gov), an international public gene expression database affiliated with NCBI and covers sufficient disease-differential genes profiling by array [16]. Selecting gene expression profile of homo sapiens in 40 patients (Female/Male:22/18) with knee OA which differ from 10 normal ones (Female/Male:6/4) with the key word "osteoarthritis" and "normal" (GEO accession: GSE51588). Download the sample series matrix file and the platform file (GPL13497) required by our study. Differentially expressed genes (DEGs) were obtained based on two criteria $(\mid \log$ FC $\mid>1$ and adj. P Value<0.05) by limma package in R software (https://www.r-project.org, ver 3.6.2). 


\subsection{Compound-disease target network}

Intersect BSHX bioactive drug targets and OA targets so as to obtain the crossing targets for compound network by Strawberry-perl-5.30.1.1 software. The compound-disease target network was built, visualized and analyzed by Cytoscape software (https://cytoscape.org, ver3.7.2). In the network, nodes indicate OA target genes and BSHX bioactive chemicals, while edges indicate interactions between them.

\subsection{Protein- protein interactions (PPI) network}

The protein-protein interactions (PPI) network was constructed by Cytoscape software based on target genes which aimed at investigating the interactions between gene regulatory proteins. Select BIP, BIOGRID, HPRD, INTACT, MINT and BIND as tool databases to construct the PPI network. To simplify the network, the topological analysis of the network was performed twice using the CytoNCA plug-in according to the screening conditions DC degree $\geq 61$ and $B C$ degree $\geq 100$.

\subsection{GO and KEGG pathway enrichment analysis}

In order to clarify the biological functions and pathways of BSHX chemicals for treating OA, Gene Ontology (GO) and Kyoto Encyclopedia of Genes and Genomes (KEGG) enrichment analysis of target genes were carried out. GO enrichment analysis was executed by Cytoscape plugin ClueGO $(\mathrm{P} \leq 0.05)$ and consists of three major categories: molecular functions $(\mathrm{MF})$, biological process $(\mathrm{BP})$ and cell chemicals (CC). Then accomplished KEGG pathway enrichment analysis $(\mathrm{P} \leq 0.05, \mathrm{Q} \leq 0.05)$ using bioconductor package in R software, set output barplot to show top 20 entries.

\subsection{Experimental validation}

A series of experiments were conducted to explore the protective effect of quercetin on interleukin-1 $\beta$-induced apoptosis of rat chondrocytes: Chondrocytes isolated from the joints of normal rats were cultured in vitro, randomly divided into 5 groups: control group (no intervention), IL-1 $\beta$ (50ng /ml), and 3 groups of quercetin $(100 \mu \mathrm{M}, 200 \mu \mathrm{M}, 400 \mu \mathrm{M}$-quercetin + IL-1 $\beta$ ). Alcian blue staining and type II collagen staining were used for observation and determination. The TUNEL 
staining method was used to observe the apoptosis of chondrocytes under a fluorescence microscope. The apoptosis rates of each group were calculated by flow cytometry, and the relative expression levels of SELE, MMP2 and COL1 were analyzed and detected by PCR and Western blot. The following antibodies were used: anti-SELE (bs-1273R, Rabbit, Bioss), anti-MMP2 (bs-0412R, Rabbit, Bioss), anti-CoL1 (bs-10423R, Rabbit, Bioss), and anti-GAPDH (60004-1-lg, Mouse, Proteintech).

\section{Results}

\subsection{Bioactive chemicals and targets in BuShenHuoXue}

A total of 1408 chemicals and 10987 targets in BSHX were collected from TCMSP. After screening by $\mathrm{OB} \geq 30 \%$ and $\mathrm{DL} \geq 0.18$, we obtained 269 bioactive chemicals and 3591 targets in all. These chemicals were identified as potential bioactive molecules for further study. The results of 269 chemicals and screening parameters of BSHX are presented in Table 2.

\subsection{Osteoarthritis-associated targets}

The dataset (GSE51588) we selected has a total of 50 samples including 10 control group(normal) samples and 40 test group (OA) samples and obtained altogether 21752 targets related to OA. We got 1591 differential genes including 950 up-regulated genes and 641 down-regulated genes after differential analysis. The top 40 differential expression genes are shown in heat map (Figure 1).

\subsection{Analysis of compound-disease target Network}

42 target genes were obtained through taking intersection of BSHX targets and 1591 OA differential genes. Combined with 104 corresponding bioactive chemicals we built a visualized compound-disease target network (Figure 2). There were 146 nodes and 208 edges shown in the network. Based on the analysis result, each compound was related to at least one gene, chemicals with the most OA targets were quercetin (MOL000098) with 22 edges and luteolin (MOL000006) with 9 edges, indicating that these chemicals might be bioactive ingredients for BSHX in the treatment of OA.

\subsection{Analysis of Protein- protein interactions (PPI) network}


The protein interactions of compound-disease target obtained from taking intersection of BSHX targets and OA differential genes were shown in PPI network. The overall network included 1229 nodes and 15419 edges, after topological analysis, we got the preliminary screening network with 124 nodes and 2358 edges, and eventually the core network with 31 nodes and 287 edges (Figure 3). Nodes in the core protein network are TP53, ESR1, HSPA8, NPM1 and VCP.

\subsection{GO and KEGG pathway enrichment analysis}

In $\mathrm{GO}$ enrichment analysis of $\mathrm{BP}, \mathrm{CC}$ and $\mathrm{MF}$, term connections were received according to $\mathrm{P}$ value $\leq 0.05$. GO enrichment analysis of target proteins is shown in Figure 4. Biological process with most significant enrichment are extracellular structure organization, muscle cell proliferation, extracellular matrix disassembly. Enriched KEGG pathways are shown in Table 3 and Figure 5,6. The major pathways for herbal intervention in OA are AGE-RAGE signaling pathway in diabetic complications (hsa04933), Relaxin signaling pathway (hsa04926), IL-17 signaling pathway (hsa04657), Tyrosine metabolism (hsa00350) and Endocrine resistance (hsa01522).Typically, figure 7 shows distribution of BSHX targets in AGE-RAGE signaling pathway in diabetic complications in KEGG.

\subsection{Experimental validation}

\subsubsection{Observation of the culture results of rat chondrocytes}

After digestion and isolation of primary cells, $80-90 \%$ of the cells were fused after 10-14 days of culture. Alcian blue staining and type II collagen staining were used for observation (Figure 8a,b ).

\section{6. 2 Quercetin can reduce the apoptosis rate of SD rat chondrocytes}

The results were observed by flow cytometry that in the control group the apoptosis rate was $1.73 \%$, and the experimental group (IL-1 $\beta$ group, the $100 \mu \mathrm{M}$ quercetin group, the $200 \mu \mathrm{M}$ quercetin group and $400 \mu \mathrm{M}$ quercetin group) of chondrocytes apoptosis rate 12.09\%, 8.78\%, $6.31 \%$, $4.31 \%$ ( Figure $9 a, b, c, d, e)$. 
The TUNEL staining method was used to observe the apoptosis of chondrocytes under a fluorescence microscope, which could be further verified more intuitively, indicating that compared with the control group, IL-1 $\beta$ can significantly increase the apoptosis of chondrocytes, while quercetin can effectively reduce the apoptosis induced by IL-1 $\beta$ (Figure 10,11 a,b,c,d,e).

\subsubsection{Quercetin decreased SELE, MMP2 and COL1 mRNA expression}

PCR results revealed the expression levels of SELE, MMP2 and COL1 genes mRNA. By taking GAPDH as internal reference, we set the relative expression rate of GAPDH as 1, and obtained the relative expression rates of the three genes in each group (Figure 12).

\subsubsection{Quercetin decreased the expression of SELE, MMP2 and COL1 protein}

By Western blotting results, it is found that the expression of the protein mediated by the genes SELE, MMP2 and COL1 are similar in trend in PCR. Also using GAPDH as internal reference, we obtained the relative expression rates of the three proteins (Figure 13, Figure 14). Western blot analysis showed that the expression of SELE, MMP2 and COL1 protein decreased significantly.

The results showed that quercetin could inhibit IL-1 $\beta$-mediated chondrocyte apoptosis when it reached a certain concentration. By inhibiting the expression of SELE, MMP2 and COL1 protein quercetin can protect the cartilage.

\section{Discussions}

$\mathrm{OA}$ is a highly prevalent degenerative joint disease of unknown etiology that involves degeneration of articular cartilage, limited intra-articular inflammation manifested by synovitis, and changes in the subchondral bone [17-21]. Due to the prevalence of obesity and an aging population, the public-health consequences of OA and OA-related disability have developed into a pressing issue, and novel therapeutic strategies are urgently required [22]. 
Osteoarthritis belongs to the category of "Bi Zheng" in terms of traditional Chinese medicine. It was first recorded in the book "Huangdi Neijing" in which the etiology and pathogenesis were described in detail. The basic pathogenesis of osteoarthritis is local qi stagnation and blood stasis in the theory of traditional Chinese medicine. TCM chemicals regulate the progression of OA by interfering with some targets in the certain signaling pathway [23]. Currently, the main etiological treatment is kidney tonifying and blood activating.

TCM preparation is to arrange the functional relationship of various herbs according to combinatorial rules of "Jun-Chen-Zuo-Shi” based on patients' syndrome, which indicates a major therapeutic chemicals combined with other agents used to restore balance of body functions and reduce toxicity such as insomnia, palpitation, infection and fever. Traditional Chinese medicine acts as a promising therapeutic option for OA. Bushenhuoxue formula is a traditional Chinese remedy that proved to be efficient for quite a long time in the first affiliated hospital of Zhejiang Chinese Medical University. Their previous researches have shown that BSHX formula could prohibit articular cartilage degradation in vivo. It was found that after 8 weeks of treatment with kidney-tonifying and bloodactivating prescription, Il-6 and MMP-13 in knee joint fluid of rabbits with knee arthritis were reduced. Their study uncovered that BSHX formula attenuates osteoarthritic cartilage degradation as a herbal MMP13 inhibitor through TGF- $\beta$ /MMP13 signaling pathway [12].

From the perspective of system biology, network pharmacology has been advocated to reinforce the established methodology for pharmacologic approach [24]. As a high throughput method, drugtarget prediction technology is cost-effective, can promptly forecast the component targets and afford basis for the application of network pharmacology in Traditional Chinese Medicine [25]. As a system biology-based methodology, it offers an applicable approach to evaluate pharmacological effects of TCM at molecular level. Moreover, targeted drugs for OA have been a great challenge for the 
development of pharmaceutical formulations [26]. Therefore, it represents a highly attractive tool owing to its potential to investigate complex relationships between chemicals and target proteins[27].

Naiqiang Zhu undertook a network pharmacology analysis of the mechanism of the effects of Shaoyao Gancao Decoction in OA, and the results suggested that SGD exerted its pharmacological effects in OA by modulating multiple pathways, including cell cycle, cell apoptosis, drug metabolism, inflammation, and immune modulation [23]. Laigen Zhang used a network pharmacology approach to elucidate the molecular mechanism governing the effect of Radix Achyranthis Bidentatae (RAB) on osteoarthritis (OA), and the results showed possible mechanisms of action for RAB include regulating the immune and inflammatory responses, reducing chondrocyte apoptosis, and protecting the joint synovial membrane and cartilage to control disease development[28]. However, no further research was conducted to verify the prediction in both two researches then reduces their persuasiveness and credibility.

In this research, a total of 104 chemicals and 42 target genes were sorted out by our screening condition, each bioactive compound acts on at least $1 \mathrm{OA}$ differential genes. Great majority of chemicals derive from licorice which indicated that licorice might be the principal effective herb of BSHX in treating OA, other herbs could play a supporting role. Quercetin and luteolin are bioactive ingredients with the most targets and they exist in multi-herb (quercetin from licorice, Lycii Fructus, Carthami Flos and Eucommiae Cortex, luteolin from Carthami Flos).

Osteoarthritis (OA) is featured by unusual extracellular matrix content (ECM) combined with articular surface erosion [29]. The precisely regulated balance of ECM synthesis, metabolism and repairment is disrupted, leading to progressive degradation of articular cartilage. Enrichment analysis of Biological Process revealed potential functions of these chemicals against OA are extracellular structure organization; muscle cell proliferation and extracellular matrix disassembly. Potential pathways are AGE-RAGE signaling pathway in diabetic complications, Relaxin signaling pathway and IL-17 signaling pathway. It is noteworthy that among the 6 target genes 
(SELE/MAPK14/MMP2/F3/COL1A1/COL3A1) in the AGE-RAGE signaling pathway in diabetic complications pathway, all of them can be interfered by quercetin, indicating that quercetin is likely to be the most promising OA treatment compound in BSHX. Quercetin was reported to exert painrelieving influence in various pain models [30] and, specially, can reduce: (i) CAR-induced mechanical hyperalgesia [30], (ii) chemotherapy-induced neuropathic pain [31], (iii) diabetic neuropathic pain [32], and (iv) muscle mechanical hyperalgesia [30]. Showing a collaborative effect and the capacity to target the oxidative stress cascade as an antioxidant, Quercetin has been employed successfully as an supplementary in vivo and experimental arthritic models $[33,34]$. Beyond this, other pathways remain to be tested to promote the treatment of OA.

\section{Conclusions}

In conclusion, TCM compound has the characteristics of multiple chemicals, multiple targets and multiple pathways in disease prevention and treatment, which are exactly what network pharmacology focus on. Our study uses network pharmacology to predict the targets and potential mechanisms of BSHX formula for OA treatment, and ultimately focuses on quercetin targeting on SELE, MMP2, and COL1 genes in BSHX formula and then inhibit the progression of OA through the AGE-RAGE signaling pathway in diabetic complications. Further experiments showed that quercetin could inhibit the apoptosis of chondrocytes induced by IL-1 $\beta$. By the mechanism of down-regulating the expression of genes involved in inflammation such as SELE, MMP2 and COL1, we made sure that quercetin can protect the cartilage. In addition, more studies are needed to reduce the toxicity of TCM and prove the rationality and compatibility of it.

In this work, we applied a novel and efficient analysis method for identification of the bioactive chemicals, crossing targets and possible molecular mechanisms of traditional Chinese herbal formulaBSHX formula. Still, there are some limitations in the present method. First, the omission of synergistic effects among ingredients in herbal formula may produce bias and incomplete results. Second, our method could not discriminate whether an ingredient could directly bind a target or just 
affect the target indirectly. Furthermore, whether quercetin can also protect cartilage and treat osteoarthritis in vivo, as well as the evaluation of its effective dose concentration, need to be further discussed and verified by corresponding experiments.

Abbreviations

OA: Osteoarthritis

BSHX: BuShenHuoXue

IL-1-: interleukin-1 $\beta$

TCMSP: Traditional Chinese Medicine Systems Pharmacology Database and Analysis Platform GEO:Gene Expression Omnibus

PPI: protein-protein interactions

GO: Gene Ontology

KEGG: Kyoto Encyclopedia of Genes and Genomes

TCM: Traditional Chinese Medicine

NP: Network pharmacology

ADME: Absorption, Distribution, Metabolism and Excretion

OB: oral bioavailability

DL: drug-likeness

GEO: Gene Expression Omnibus

Declarations

Ethics approval and consent to participate: Not applicable

Consent for publication: The manuscript is approved by all authors for publication.

Availability of data and materials: The datasets used or analysed during the current study are available from the corresponding author on reasonable request.

Competing interests: The authors declare that they have no competing interests.

Funding: Not applicable 
Authors' contributions: Auther Xiong Wen contributed to acquisition of data, or analysis and interpretation of data, drafting the article. Auther Cai Xianhua contributed to the conception and design of the study, revising it critically for important intellectual content, final approval of the version to be published.

Acknowledgements: We gratefully acknowledge the contributions of Helin Feng (Department of Orthopedics, The Fourth Hospital of Hebei Medical University) in the study design and data analysis Authors' information (optional): Not applicable

References:

1. Gu YT, Chen J, Meng ZL, et al. Research progress on osteoarthritis treatment mechanisms. Biomed Pharmacother. 2017; 93:1246-1252. DOI: 10.1016/j.biopha.2017.07.034

2. Thomas AC, Hubbard-Turner T, Wikstrom EA, et al. Epidemiology of Posttraumatic Osteoarthritis. J Athl Train. 2017;52(6):491-496. DOI: 10.4085/1062-6050-51.5.08

3. Li MH, Xiao R, Li JB, et al. Regenerative approaches for cartilage repair in the treatment of osteoarthritis. Osteoarthritis Cartilage. 2017;25(10):1577-1587. DOI: 10.1016/j.joca.2017.07.004

4. Hermann W, Lambova S, Muller-Ladner U. Current Treatment Options for Osteoarthritis. Curr Rheumatol Rev. 2018;14(2):108-116. DOI: 10.2174/1573397113666170829155149

5. Tao W, Luo X, Cui B, et al. Practice of traditional Chinese medicine for psycho-behavioral intervention improves quality of life in cancer patients: A systematic review and meta-analysis. Oncotarget. 2015;6(37):39725-39739. DOI: 10.18632/oncotarget.5388

6. Hao P, Jiang F, Cheng J, et al. Traditional Chinese Medicine for Cardiovascular Disease: Evidence and Potential Mechanisms. J Am Coll Cardiol. 2017 Jun 20;69(24):2952-2966. DOI: 10.1016/j.jacc.2017.04.041

7. Zhu F, Yin L, Ji L, et al. Suppressive effect of Sanmiao formula on experimental gouty arthritis by inhibiting cartilage matrix degradation: An In Vivo and In Vitro Study. Int Immunopharmacol. 2016 30:36-42. DOI: 10.1016/j.intimp.2015.11.010 
8. Wang F, Shi L, Zhang Y, et al. A Traditional Herbal Formula Xianlinggubao for Pain Control and Function Improvement in Patients with Knee and Hand Osteoarthritis: A Multicenter, Randomized, Open-Label, Controlled Trial. Evid Based Complement Alternat Med. 2018 Feb 12; 2018:1827528. DOI: $10.1155 / 2018 / 1827528$

9. Li L, Liu H, Shi W. et al. Insights into the action mechanisms of traditional Chinese medicine in osteoarthritis. Evid Based Complement Alternat Med. 2017:5190986. DOI: 10.1155/2017/5190986

10. Wang H, Shen Z, Zeng J. Clinical observation of nourishing kidney and activating blood therapy for degenerative osteoarthritis. Journal of New Chinese Medicine, 2017 April Vol.49 No.4:65-67. DOI: $10.13457 /$ j.cnki.jncm.2017.04.022

11.Zheng $\mathrm{T}, \mathrm{Xu} \mathrm{ZG}, \mathrm{Xu} \mathrm{ZY}$, et al. Clinical Effect of Bushen Huoxue Prescription on Knee Osteoarthritis. Chinese archives of traditional chinese medicine, 2019 Jun Vol. 37 No. 6:15061509. DOI: 10. 13193 / j. issn. 1673-7717. 2019. 06. 058

12.Wang PE, Zhang L, Ying J,et al. Bushenhuoxue formula attenuates cartilage degeneration in an osteoarthritic mouse model through TGF-beta/MMP13 signaling. J Trans1 Med. 2018 16(1):72. DOI: $10.1186 / \mathrm{s} 12967-018-1437-3$

13. Zhang R, Zhu X, Bai H, et al. Network Pharmacology Databases for Traditional Chinese Medicine: Review and Assessment. Front Pharmacol. 2019 Feb 21; 10:123. DOI: 10.3389/fphar.2019.00123

14. Lee WY, Lee CY, Kim YS, et al. The Methodological Trends of Traditional Herbal Medicine Employing Network Pharmacology. Biomolecules. 2019 Aug 13;9(8).362. DOI: 10.3390/biom9080362

15. Yang H, Li Y, Shen S, et al. Network Pharmacology-Based Investigation into the Mechanisms of Quyushengxin Formula for the Treatment of Ulcerative Colitis. Evid Based Complement Alternat Med. 2019 Dec 20; 2019:7870424. DOI: 10.1155/2019/7870424

16. Barrett T, Troup DB, Wilhite SE, et al. NCBI GEO: archive for functional genomics data sets--10 years on. Nucleic Acids Res. 2011;39(Database issue): D1005-1010. DOI: 10.1093/nar/gkq1184 
17.Pottie P, Presle N, Terlain B, et al. Obesity and osteoarthritis: more complex than predicted! Ann Rheum Dis. 2006;65(11):1403-1405. DOI:10.1136/ard.2006.061994

18. Goldring MB, Marcu KB. Cartilage homeostasis in health and rheumatic diseases. Arthritis Res Ther. 2009;11(3):224. DOI: 10.1186/ar2592

19. Shamoon M, Hochberg MC. Treatment of osteoarthritis with acetaminophen: efficacy, safety, and comparison with nonsteroidal anti-inflammatory drugs. Curr Rheumatol Rep. 2000;2(6):454-458. DOI:10.1007/s11926-000-0020-z

20. Alcaraz MJ, Megías J, García-Arnandis I, et al. New molecular targets for the treatment of osteoarthritis. Biochem Pharmacol. 2010;80(1):13-21. DOI: 10.1016/j.bcp.2010.02.017

21. Yan K, Shen Y. Aliskiren has chondroprotective efficacy in a rat model of osteoarthritis through suppression of the local renin-angiotensin system. Mol Med Rep. 2017 16(4): 3965-3973. DOI: $10.3892 / \mathrm{mmr} .2017 .7110$

22. Umlauf D, Frank S, Pap T et al. Cartilage biology, pathology, and repair. Cell Mol Life Sci. 2010;67(24):4197-4211. DOI: 10.1007/s00018-010-0498-0

23. Zhu N, Hou J, Ma G, et al. Network Pharmacology Identifies the Mechanisms of Action of Shaoyao Gancao Decoction in the Treatment of Osteoarthritis. Med Sci Monit. 2019;14;25:6051-6073. DOI: 10.12659/MSM.915821

24. Hopkins AL. Network pharmacology. Nat Biotechnol. 2007;25(10):1110-1111. DOI:10.1038/nbt1007-1110

25. Wu CW, Lu L, Liang SW, et al. Application of drug-target prediction technology in network pharmacology of traditional Chinese medicine. Zhongguo Zhong Yao Za Zhi. 2016;41(3):377-382. DOI: $10.4268 / \mathrm{cjcmm} 20160303$

26. Hathout RM, El-Ahmady SH, Metwally AA. Curcumin or bisdemethoxycurcumin for nose-tobrain treatment of Alzheimer disease? A bio/chemo-informatics case study. Nat Prod Res. 2018;32(24):2873-2881. DOI: 10.1080/14786419.2017.1385017 
27. Yang M, Chen J, Xu L, et al. A Network Pharmacology Approach to Uncover the Molecular Mechanisms of Herbal Formula Ban-Xia-Xie-Xin-Tang. Evid Based Complement Alternat Med. 2018;2018:4050714. Published 2018 Oct 16. DOI:10.1155/2018/4050714.

28. Zhang L, Shi X, Huang Z, et al. Network Pharmacology Approach to Uncover the Mechanism Governing the Effect of Radix Achyranthis Bidentatae on Osteoarthritis. BMC Complement Med Ther. 2020;20(1):121. Published 2020 Apr 21. DOI:10.1186/s12906-020-02909-4.

29. Bomer N, den Hollander W, Ramos YF, et al. Translating genomics into mechanisms of disease: osteoarthritis. Best Pract Res Clin Rheumatol. 2015;29(6):683-91. DOI: 10.1016/j.berh.2016.01.001.

30. Carullo G, Cappello AR, Frattaruolo L, et al. Quercetin and derivatives: useful tools in inflammation and pain management. Future Med Chem. 2017;9(1):79-93. DOI: 10.4155/fmc2016-0186.

31. Gao W, Zan Y, Wang ZJ, et al. Quercetin ameliorates paclitaxel-induced neuropathic pain by stabilizing mast cells, and subsequently blocking PKCe-dependent activation of TRPV1. Acta Pharmacol Sin. 2016;37(9):1166-77. DOI: 10.1038/aps.2016.58.

32. Borghi SM, Pinho-Ribeiro FA, Fattori V, et al. Quercetin inhibits peripheral and spinal cord nociceptive mechanisms to reduce intense acute swimming-induced muscle pain in mice. PLoS One. 2016 Sep 1;11(9): e0162267. DOI: 10.1371/journal.pone.0162267.

33. Britti D, Crupi R, Impellizzeri D, et al. A novel composite formulation of palmitoylethanolamide and quercetin decreases inflammation and relieves pain in inflammatory and osteoarthritic pain models. BMC Vet Res. 2017 Aug 2;13(1):229. DOI: 10.1186/s12917-017-1151-z.

34. Petrosino S, Di Marzo V. The pharmacology of palmitoylethanolamide and first data on the therapeutic efficacy of some of its new formulations. Br J Pharmacol. 2017 Jun;174(11):13491365. DOI: $10.1111 / \mathrm{bph} .13580$. 
Figure 1: The top 40 up-regulated and down-regulated differential expression genes between normal tissues and OA tissues.

Figure 2: The network of 104 active chemicals and 42 crossing targets of BSHX and OA. In the left circle, the node color of chemicals reflects different herb sources, green elliptic nodes derive from Gancao, pink from Duzhong, orange from Honghua, purple from Fuzi, gray from Shanyao, yellow from Gouqizi, brown from Shanzhuyu, gold from Taoren, and red from multi-herb. In the right circle, blue elliptic nodes derive from genes.

Figure 3: The PPI network of crossing genes of BSHX against OA. In the network diagram, node represents the molecular target protein, and edge represents the relationship between the components and the target.

Figure 4: GO enrichment analysis of target proteins. The number of GO entries in the functional categories of cell composition, molecular function, and biological process (FDR $<0.05$ ).

Figure 5: Enriched KEGG pathways and the associated targets.

Figure 6: Enriched KEGG pathways and the associated targets. 
Figure 7: Distribution of BSHX targets in AGE-RAGE signaling pathway in diabetic complications in KEGG, red nodes represents BSHX targets and white nodes represents other genes in the pathway.

Figure 8a: Alcian blue stain of chondrocytes

Figure 8b: Type II collagen staining of chondrocytes

Figure 9a,b,c,d,e: Flow cytometry analysis of apoptosis in five groups of chondrocytes (control, IL-1 $\beta, 100 \mu \mathrm{M}$ quercetin, $200 \mu \mathrm{M}$ quercetin, $400 \mu \mathrm{M}$ quercetin )

Figure 10a,b,c,d,e: Apoptosis of chondrocytes detected by TUNEL staining (control, IL-1 $\beta, 100 \mu$ M quercetin, $200 \mu \mathrm{M}$ quercetin, $400 \mu \mathrm{M}$ quercetin )

Figure 11a,b,c,d,e: Apoptosis of chondrocytes detected by TUNEL and DAPI staining (control, IL-1 $\beta, 100 \mu \mathrm{M}$ quercetin, $200 \mu \mathrm{M}$ quercetin, $400 \mu \mathrm{M}$ quercetin)

Figure 12 a,b,c: Relative gene expression rates of SELE, MMP2 and COL1 in five groups of chondrocytes (internal reference of GAPDH)

Figure 13: Relative expression rates of SELE, MMP2 and COL1 protein in five groups of chondrocytes (internal reference of GAPDH)

Figure 14: Electrophoretic images of expressions of SELE, MMP2, COL1 and GAPDH in chondrocytes of five groups 
Figures

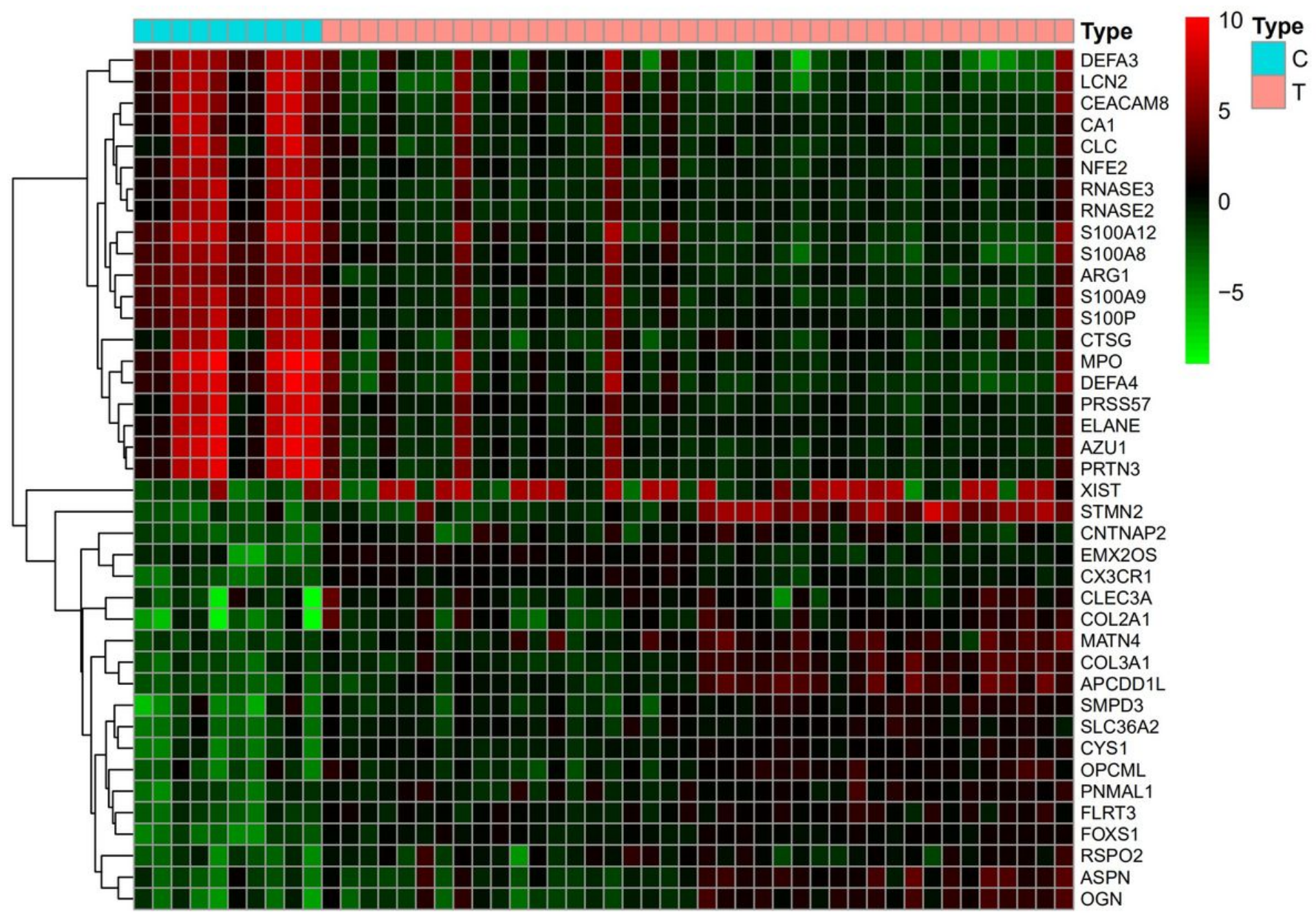

Figure 1

The top 40 up-regulated and down-regulated differential expression genes between normal tissues and OA tissues. 


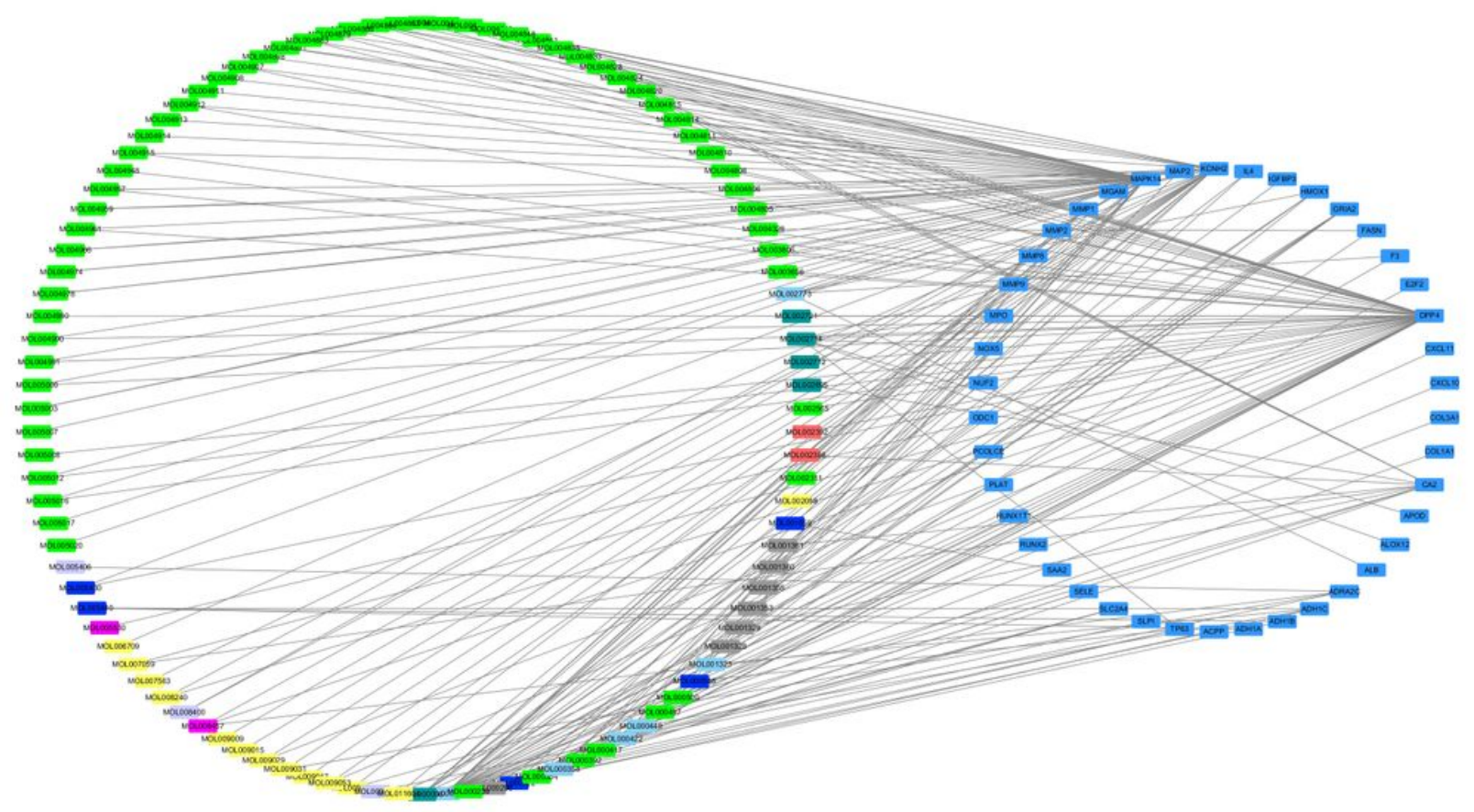

\section{Figure 2}

The network of 104 active chemicals and 42 crossing targets of BSHX and OA. In the left circle, the node color of chemicals reflects different herb sources, green elliptic nodes derive from Gancao, pink from Duzhong, orange from Honghua, purple from Fuzi, gray from Shanyao, yellow from Gouqizi, brown from Shanzhuyu, gold from Taoren, and red from multi-herb. In the right circle, blue elliptic nodes derive from genes.
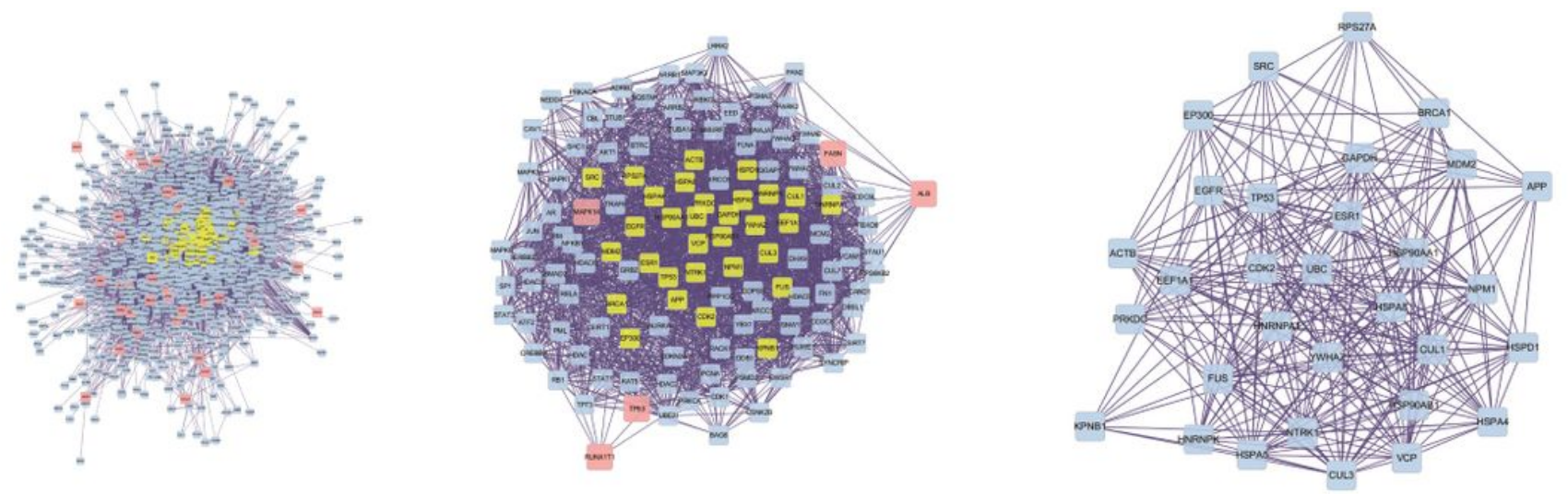

\section{Figure 3}

The PPI network of crossing genes of BSHX against OA. In the network diagram, node represents the molecular target protein, and edge represents the relationship between the components and the target. 

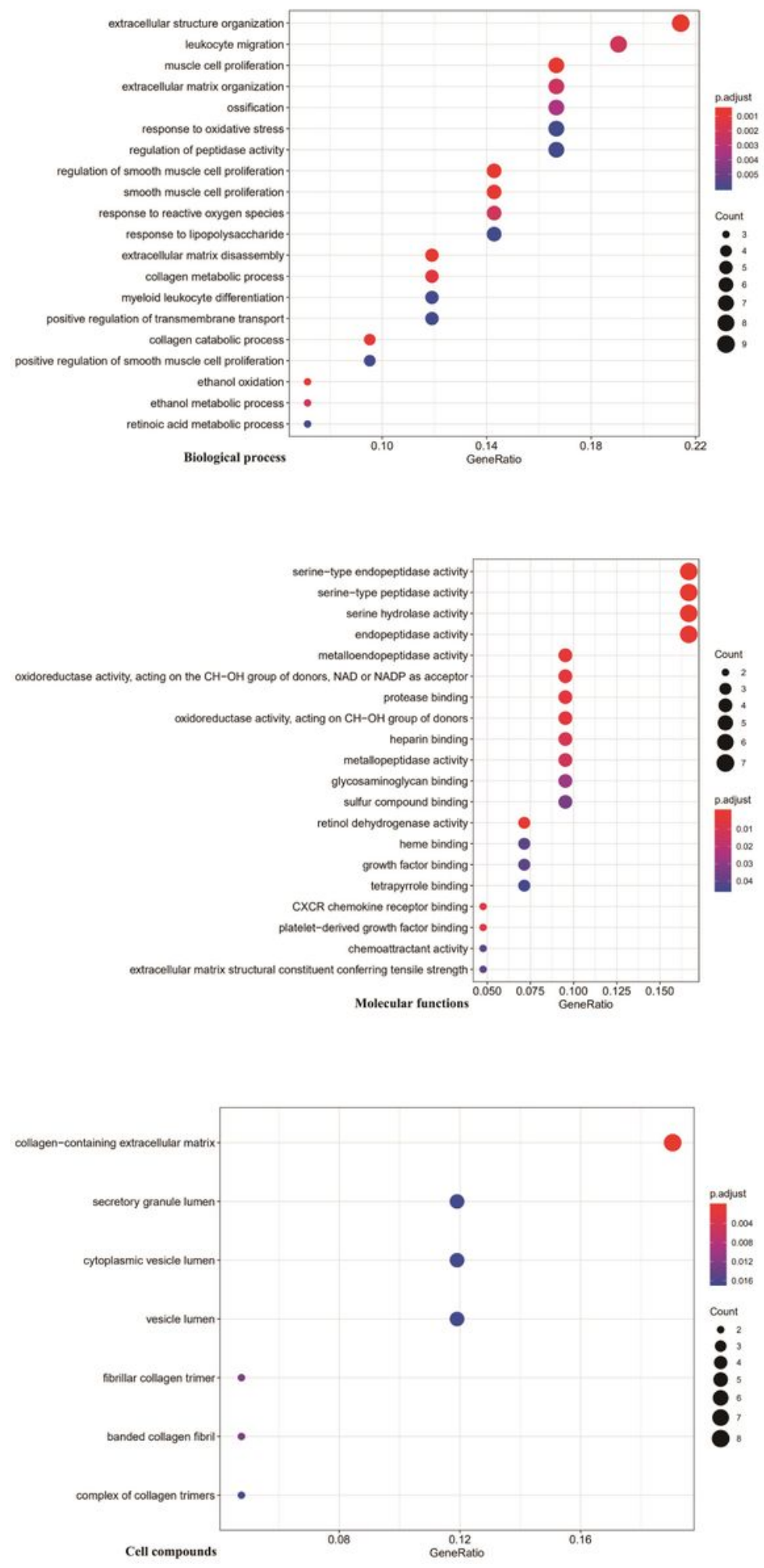

\section{Figure 4}

$\mathrm{GO}$ enrichment analysis of target proteins. The number of GO entries in the functional categories of cell composition, molecular function, and biological process (FDR $<0.05)$. 


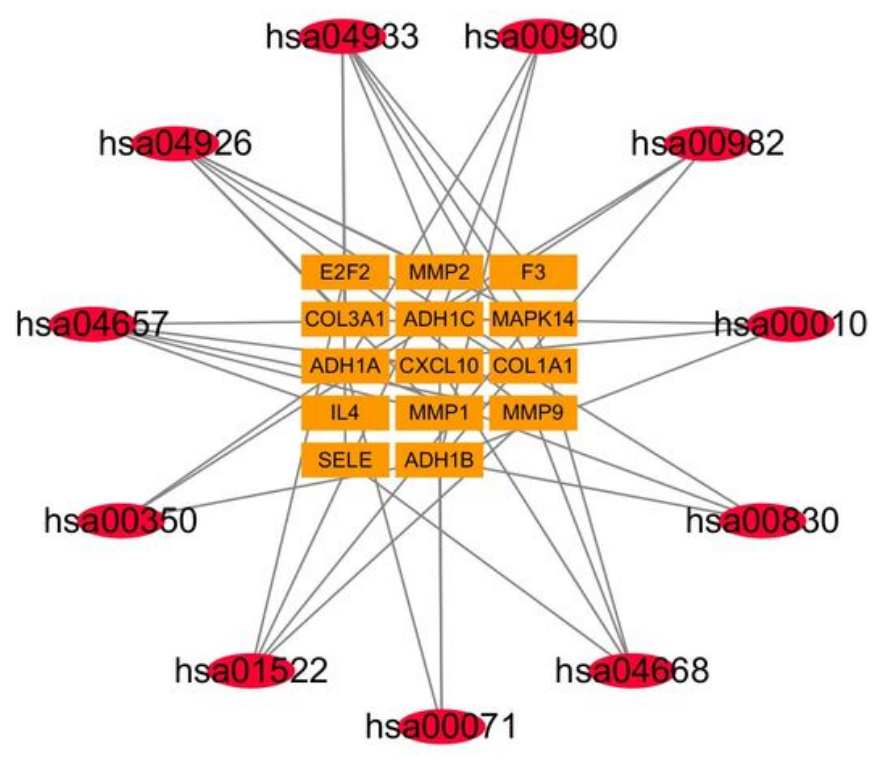

\section{Figure 5}

Enriched KEGG pathways and the associated targets.

AGE-RAGE signaling pathway in diabetic complications -

Relaxin signaling pathway

IL-17 signaling pathway

Tyrosine metabolism

Endocrine resistance

Fatty acid degradation

TNF signaling pathway

Retinol metabolism

Glycolysis / Gluconeogenesis

Drug metabolism - cytochrome P450

Metabolism of xenobiotics by cytochrome P450

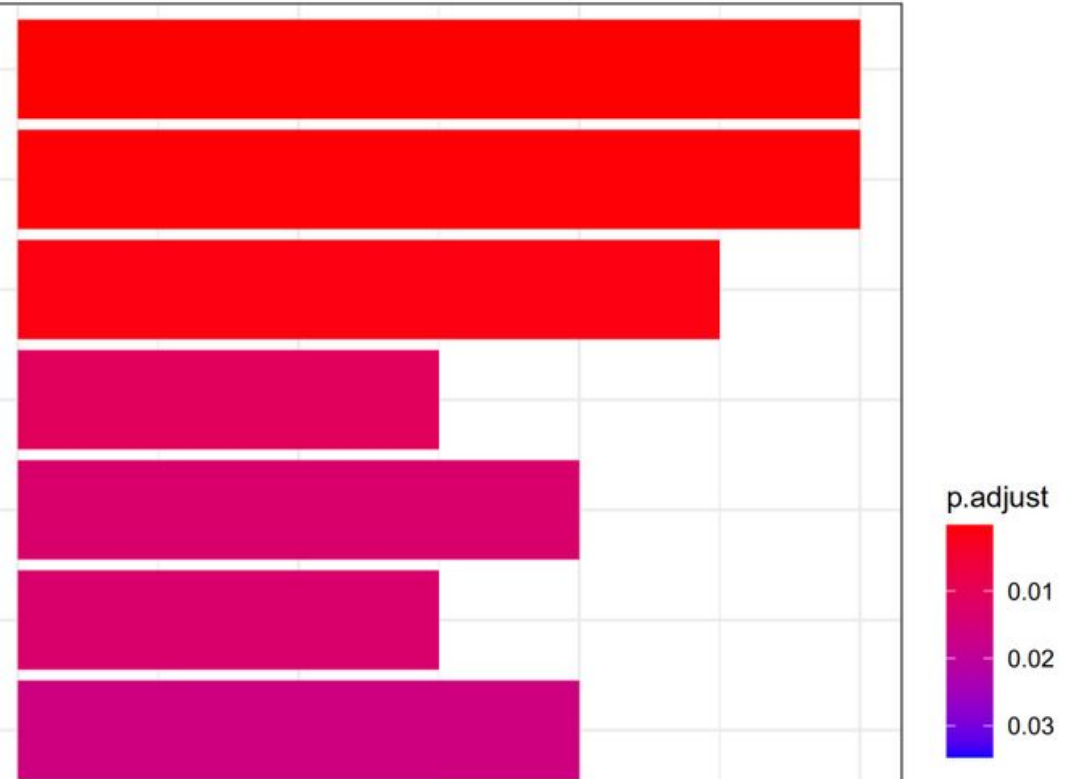

\section{Figure 6}


Enriched KEGG pathways and the associated targets.

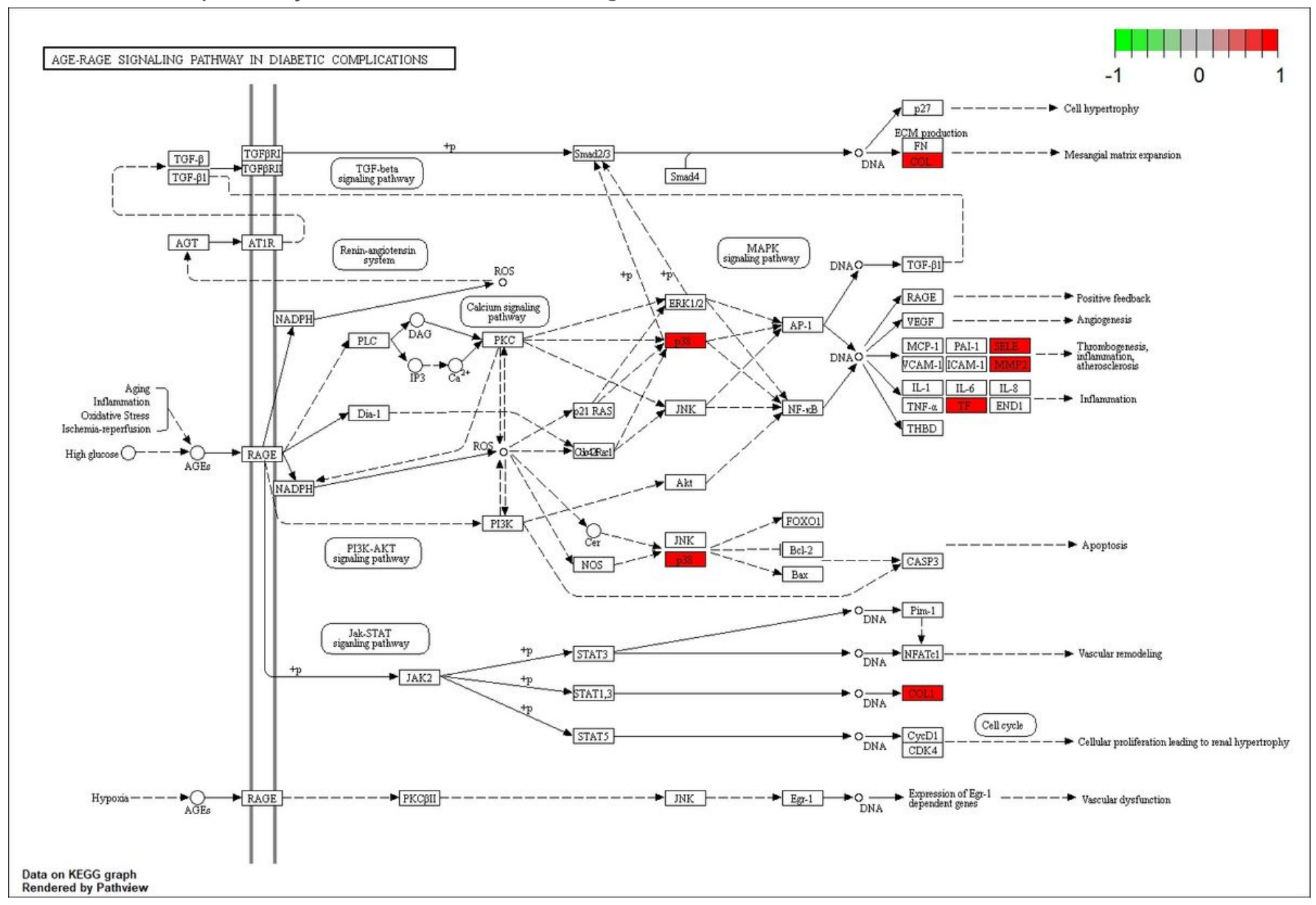

Figure 7

Distribution of BSHX targets in AGE-RAGE signaling pathway in diabetic complications in KEGG, red nodes represents BSHX targets and white nodes represents other genes in the pathway.

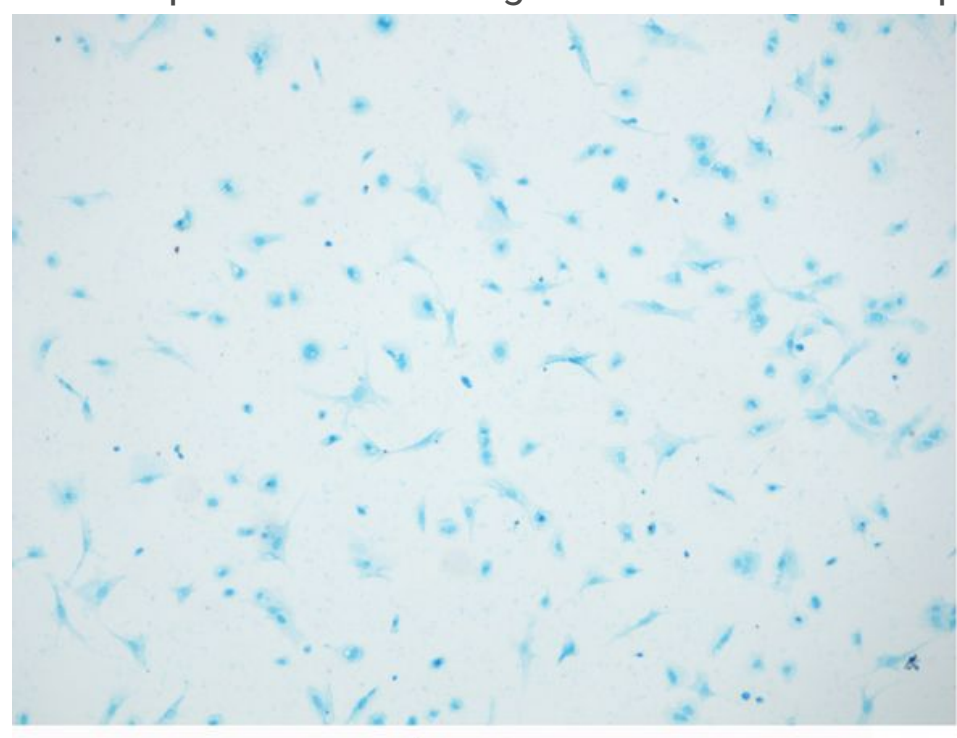

Figure 8a

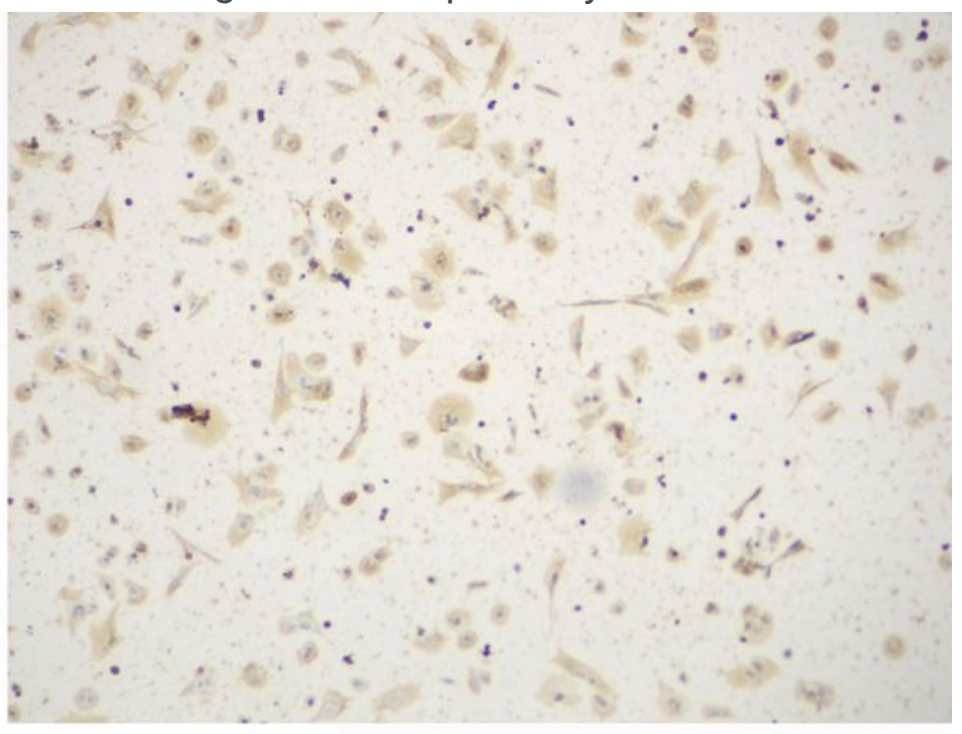

Figure $8 b$ 
Figure 8

Figure 8a: Alcian blue stain of chondrocytes Figure 8b: Type II collagen staining of chondrocytes

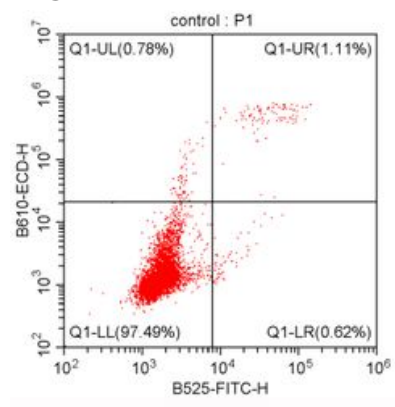

Figure 9a

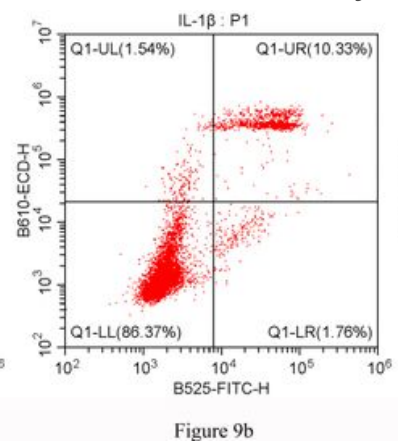

Figure 9b

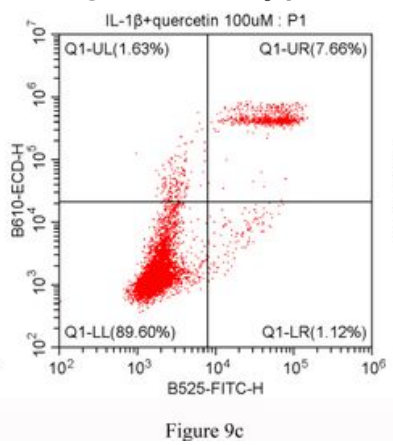

Figure 9c

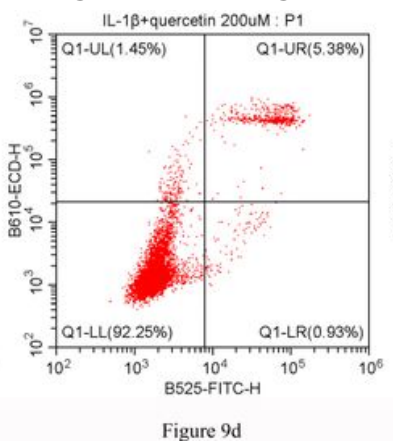

Figure 9d

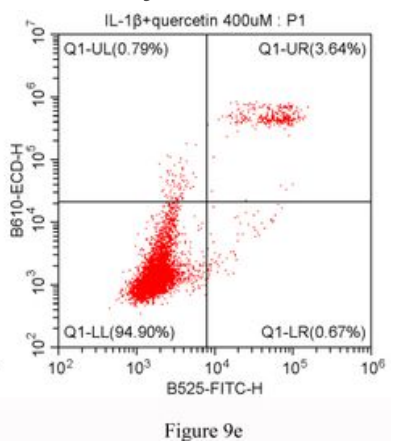

Figure 9e

\section{Figure 9}

Figure 9 a,b,c,d,e: Flow cytometry analysis of apoptosis in five groups of chondrocytes $\llbracket$ control, IL-1 $\beta$, $100 \mu \mathrm{M}$ quercetin, $200 \mu \mathrm{M}$ quercetin, $400 \mu \mathrm{M}$ quercetin $\otimes$

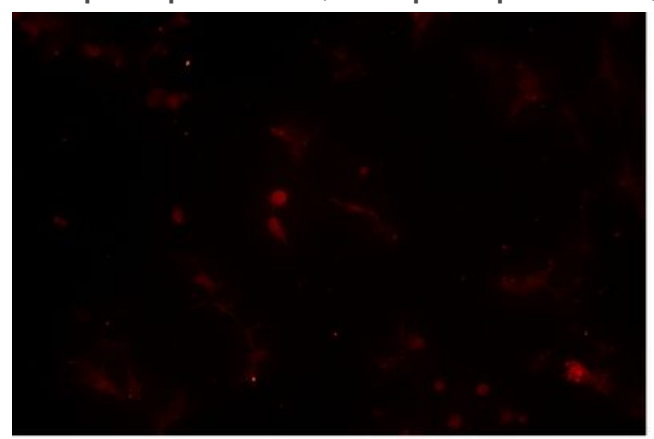

Figure 10a

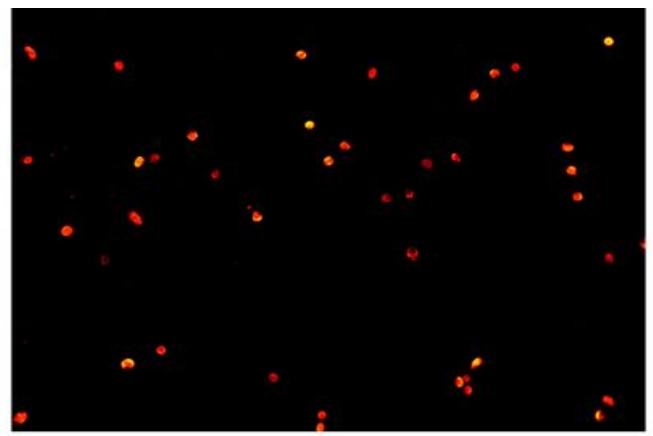

Figure 10b

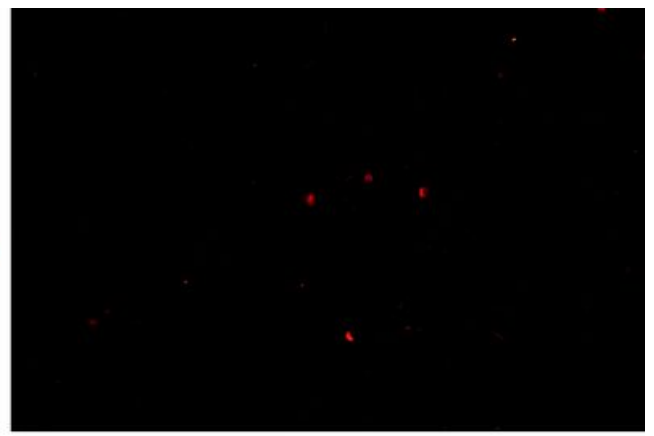

Figure 10c

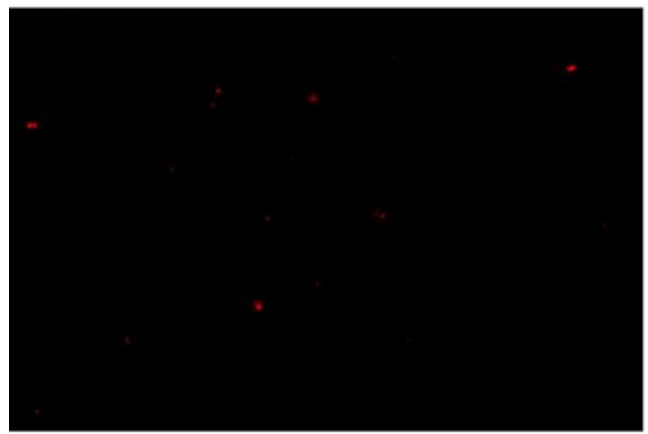

Figure 10d

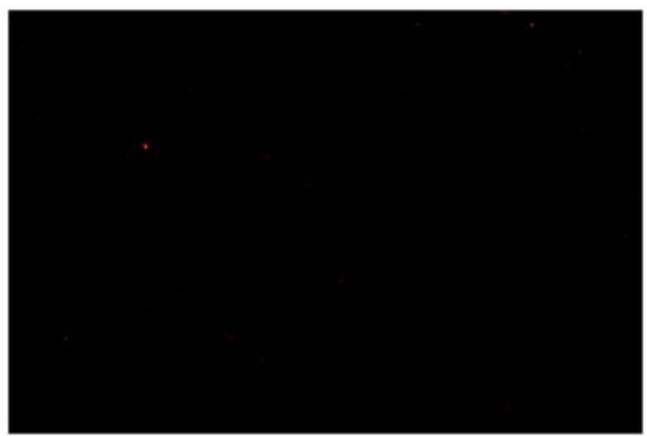

Figure 10e

\section{Figure 10}

Figure 10a,b,c,d,e: Apoptosis of chondrocytes detected by TUNEL staining $₫$ control, IL-1 $\beta, 100 \mu \mathrm{M}$ quercetin, $200 \mu \mathrm{M}$ quercetin, $400 \mu \mathrm{M}$ quercetin $\nabla$ 


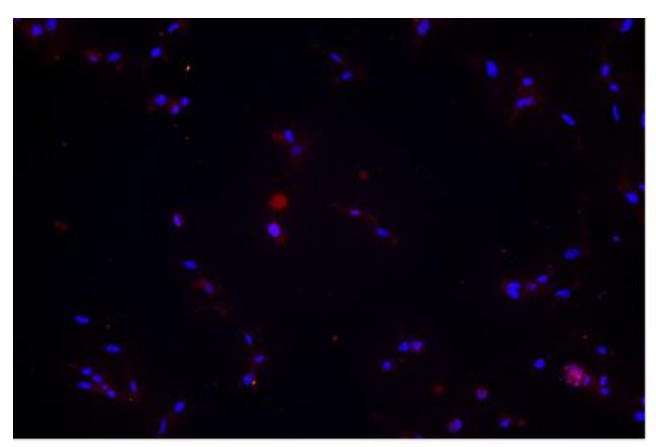

Figure 11a

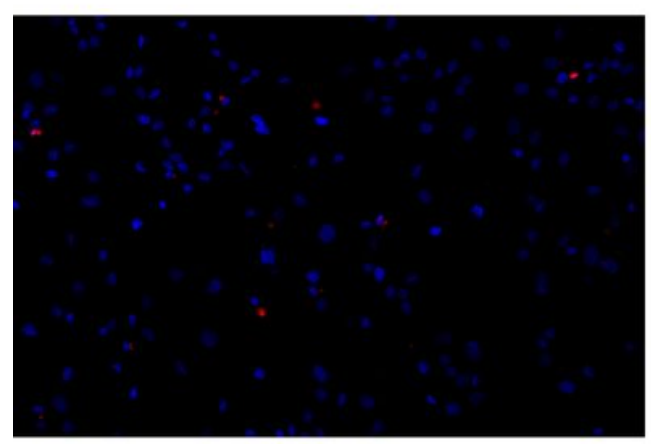

Figure 11d

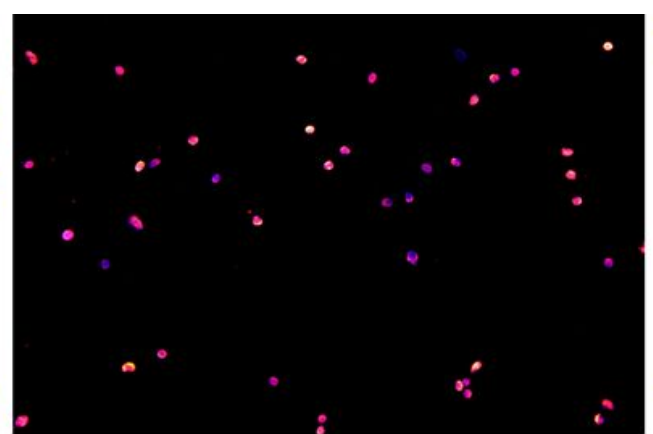

Figure 11b

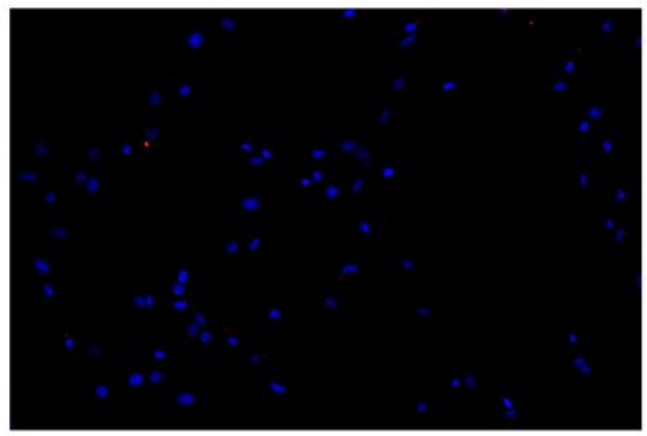

Figure 11e
Figure 11c

\section{Figure 11}

Figure 11 a,b,c,d,e: Apoptosis of chondrocytes detected by TUNEL and DAPI staining $₫$ control, IL-1 $\beta, 100 \mu M$ quercetin, $200 \mu \mathrm{M}$ quercetin, $400 \mu \mathrm{M}$ quercetin $\otimes$
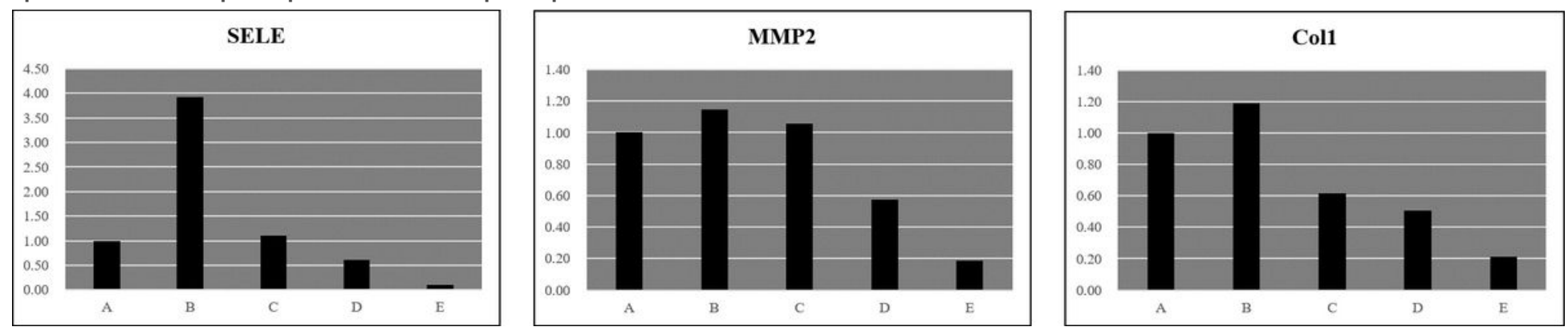

\section{Figure 12}

Figure $12 \mathrm{a}, \mathrm{b}, \mathrm{c}$ : Relative gene expression rates of SELE, MMP2 and COL1 in five groups of chondrocytes (internal reference of GAPDH)
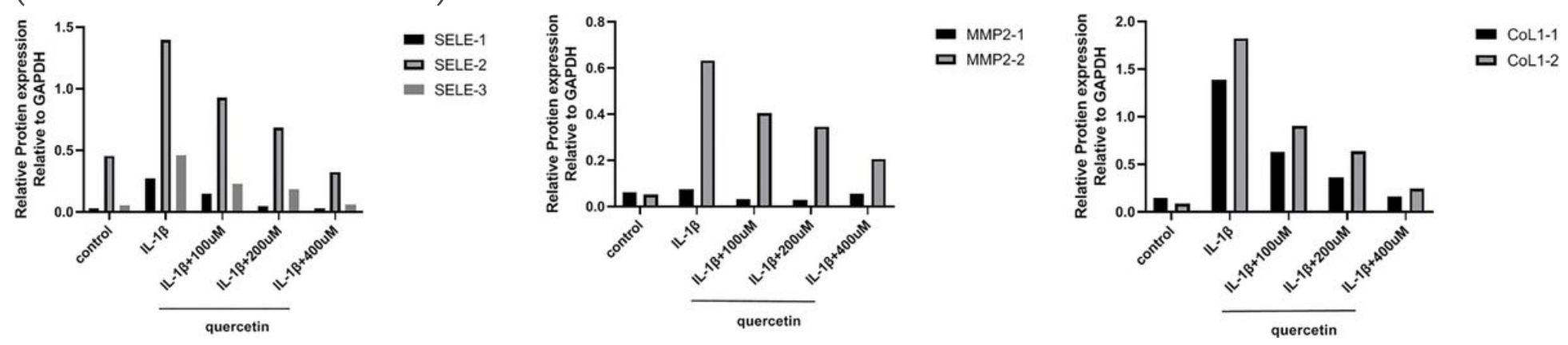

Figure 13 
Figure 13: Relative expression rates of SELE, MMP2 and COL1 protein in five groups of chondrocytes (internal reference of GAPDH)

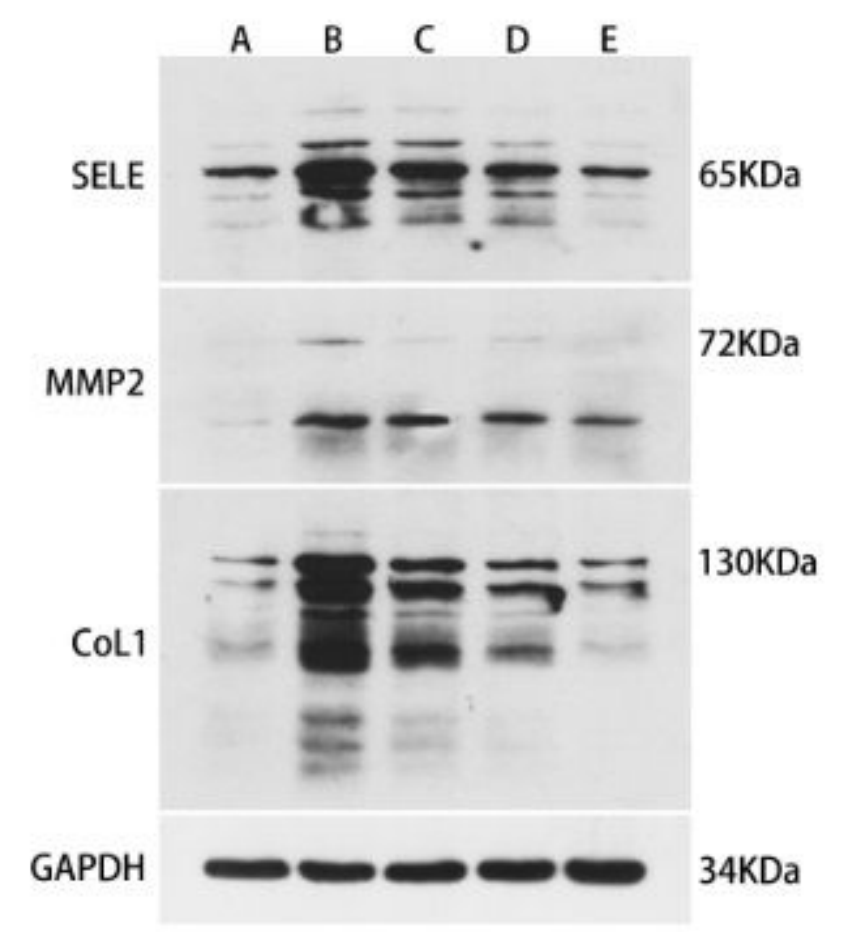

Figure 14

Figure 14: Electrophoretic images of expressions of SELE, MMP2, COL1 and GAPDH in chondrocytes of five groups

\section{Supplementary Files}

This is a list of supplementary files associated with this preprint. Click to download.

- Table1.pdf 